\title{
Managing Extremity Soft Tissue Sarcoma Patients: Surgery Alone or in Combination with Radiotherapy? An Editorial on the Paper by Fiore et al.
}

\author{
Rick Haas, $\mathrm{MD}, \mathbf{P h D}^{1,2}$ \\ ${ }^{1}$ Department of Radiotherapy, The Netherlands Cancer Institute, Amsterdam, The Netherlands; ${ }^{2}$ Department of \\ Radiotherapy, Leiden University Medical Center, Leiden, The Netherlands
}

Dr Fiore and colleagues are to be congratulated on their analysis of local control probability after surgery alone in high-risk extremity soft tissue sarcomas (ESTSs). ${ }^{1}$ Although both national comprehensive cancer network $(\mathrm{NCCN})^{2}$ and European society for medical oncology $(\mathrm{ESMO})^{3}$ guidelines suggest combining limb salvage surgery with radiotherapy (RT), and although no data are currently available from randomized studies suggesting to refrain from RT, the results from the study by Dr Fiore et al. are remarkable in several ways, but also need to be considered in the right perspective.

\section{WHAT ARE THE REMARKABLE FINDINGS?}

There were less R0 resections $(69.4 \%$ vs. $78.9 \%$; $p=0.182$ ) in patients in group B (without RT) compared with those in group A, yet local control in both groups was comparable. What one would expect is that, specifically in a subgroup with R1 resections, perioperative RT would compensate for these microscopically positive margins with respect to local control, which it did not $-66.3 \%$ vs. $72.1 \%$.

The local failure rate without RT in R1 resections was 27.9\%. As found by Alektiar (manuscript reference \#24) and many others, radiation would decrease the local failure rate with a factor of $2-3$, mounting to a failure rate of 9-14\%, and therefore a local control estimate of $86-91 \%$.

(C) Society of Surgical Oncology 2018

First Received: 8 February 2018;

Published Online: 27 February 2018

R. Haas, MD, $\mathrm{PhD}$

e-mail: r.haas@nki.nl
This rate is comparable with the literature; however, in the study by Fiore et al., it is unexpectedly lower at $66.3 \%$.

The local failure rate without RT in R0 resections was fairly low at $14.8 \%$. If, again, the risk on local relapse was reduced with a factor of $2-3$, a local failure rate of $5-8 \%$ would be expected, leading to a local control estimate of 92-95\%; in the study by Fiore et al. the rate was $88 \%$. In addition, these rates are comparable with the literature, specifically pertaining to the data obtained by O'Sullivan et al. in the Canadian randomized SR-2 trial. ${ }^{4}$ What remains unexplained (except for selection biases on histology and the performance of isolated limb perfusions) is the observation that, in their setting, the addition of RT to limb-sparing surgery led to so little gain in local control, which is contradictory to many other series. ${ }^{4,5}$ Furthermore, the definition of resection margins may differ across institutes. Gundle et al. recently proposed a clinically meaningful classification system that may serve as a standardization method. ${ }^{6}$ Another issue to consider more indepth is the timing of RT. The data are reported by Fiore et al., and, for overview, have been summarized in Table 1 below. RT was predominantly administered postoperatively, and as such it did not increase local control compared with surgery alone. However, when performed preoperatively, the local control rate increased by an absolute gain of $3.9 \%$ (in cases of R1) to $7.8 \%$ (in cases of $\mathrm{R} 0$ ).

A reasonable interpretation of these data with respect to surgery alone in high-risk ESTSs could be that referral to highly experienced sarcoma surgeons translates into high local control rates, even when selectively (not routinely) refraining from perioperative RT. This observation has also been reported by Blay et al. ${ }^{7}$ suggesting significantly more $\mathrm{R} 0$ and less R2 resections, less reoperations, and higher 
TABLE 1 Local control rates in different subgroups in the Fiore et al. study

\begin{tabular}{|c|c|c|c|c|c|c|c|}
\hline & No RT, group B & With RT, group & & & & & \\
\hline & & All perioperative & & Preoperati & & Postoperati & \\
\hline & {$[n=72]$} & {$[n=318]$} & $p$ value & {$[n=107]$} & Absolute gain & {$[n=211]$} & Absolute gain \\
\hline R0 & $85.2 \%[n=50]$ & $88 \%[n=251]$ & 0.240 & $93 \%$ & $+7.8 \%$ & $87 \%$ & $+1.8 \%$ \\
\hline $\mathrm{R} 1$ & $72.1 \%[n=22]$ & $66.3 \%[n=66]$ & 0.730 & $76 \%$ & $+3.9 \%$ & $66 \%$ & $-6.1 \%$ \\
\hline
\end{tabular}

Note for group A: $251+66=317$, there was one other case with an R2 resection

$R T$ radiotherapy

relapse-free survival (RFS) and local RFS in reference centers.

Studying wound complications is yet another elaboration on surgical skills and the obvious need to have sarcoma patients treated at reference centers. Several series suggest a wound complication rate after surgery alone of approximately $17 \%$, but a doubling to $35 \%$ if surgery was preceded by preoperative RT. ${ }^{4,8}$ Although the specific criteria for registering a wound complication are not described, Dr Fiore and co-workers recorded a substantially lower rate of $13.6 \%$ overall. Somewhat surprising is that this rate was $20.5 \%$ in the group without RT and $11.9 \%$ in the group with perioperative radiation, which one would have expected to be the other way around. However, this paradox can be explained by the selection biases of patients not undergoing RT, as described in Table 2 of the Fiore et al. study. Approximately two-thirds of the reasons not to apply radiation had to do with (the anticipation of) complications, and therefore the radiation abstention was an individualized approach. ${ }^{9}$

The take home messages from this important article by Fiore et al. could be as follows.

- Referral to experienced sarcoma surgeons may lead to higher local control rates after limb-sparing surgery alone than would be expected from other reports (see the studies by Fiore et al. and Gundle et al. ${ }^{6}$ ).

- Referral to experienced sarcoma surgeons may lead to lower wound complication rates (see the studies by Fiore et al. and O'Sullivan et al. ${ }^{4}$ ).

- When RT is offered, the absolute gain in local control may be higher after preoperative RT compared with postoperative RT, irrespective of resection margin status (see the studies by Fiore et al. and Sampath et al. ${ }^{10}$ )

- Obviously, the addition of RT to increase local control rates compared with surgery alone comes at the cost of a toxicity profile that differs between preoperative (wound complications) and postoperative RT (permanent late functional deficits). ${ }^{411}$ The decision on prescribing RT should not be taken lightly and is best left to experienced multidisciplinary teams. Having stated that, until well-designed, randomized studies on the additional value of RT, as well as RT dose, so dictate (e.g. NCT00870701, NCT02565498), standard management of high-risk ESTSs remains a combination of limb-sparing surgery and RT., ${ }^{2,3}$

\section{REFERENCES}

1. Fiore M, Ford S, Callegaro D, et al. Adequate local control in high-risk soft tissue sarcoma of the extremity treated with surgery alone at a reference centre: should radiotherapy still be a standard? Ann Surg Oncol. 2018. https://doi.org/10.1245/s10434-0186393-x.

2. von Mehren M, Randall RL, Benjamin RS, et al. National comprehensive cancer network. Soft tissue sarcoma, version 2.2016. $J$ Natl Compr Canc Netw. 2016;14:758-86.

3. The ESMO/European Sarcoma Network Working Group. Soft tissue and visceral sarcomas: ESMO Clinical Practice Guidelines for diagnosis, treatment and follow-up. Ann Oncol. 2014;25:iii102-12.

4. O'Sullivan B, Davis AM, Turcotte R, et al. Preoperative versus postoperative radiotherapy in soft-tissue sarcoma of the limbs: a randomised trial. Lancet. 2002;359:2235-41.

5. Beane JD, Yang JC, White D, et al. Efficacy of adjuvant radiation therapy in the treatment of soft tissue sarcoma of the extremity: 20-year follow-up of a randomized prospective trial. Ann Surg Oncol. 2014;21:2484-89.

6. Gundle KR, Kafchinski L, Gupta S et al. analysis of margin classification systems for assessing the risk of local recurrence after soft tissue sarcoma resection. J Clin Oncol. 2018. https:// doi.org/10.1200/jco.2017.74.6941.

7. Blay JY, Soibinet P, Penel $\mathrm{N}$ et al. Improved survival using specialized multidisciplinary board in sarcoma patients. Ann Oncol. 2017;28:2852-59.

8. Cannon CP, Ballo MT, Zagars GK et al. Complications of combined modality treatment of primary lower extremity softtissue sarcomas. Cancer. 2006;107:2455-61.

9. Gronchi A. Individualizing the use/non-use of radiation therapy (RT) in soft tissue sarcoma (STS): When abstention is better than care. J Surg Oncol. 2015;111:133-4.

10. Sampath S, Schultheiss TE, Hitchcock YJ et al. Preoperative versus postoperative radiotherapy in soft-tissue sarcoma: multiinstitutional analysis of 821 patients. Int J Radiat Oncol Biol Phys. 2011;81:498-505.

11. Davis AM, O'Sullivan B, Turcotte R et al. Late radiation morbidity following randomization to preoperative versus postoperative radiotherapy in extremity soft tissue sarcoma. $R a$ diother Oncol. 2005;75:48-53. 